\title{
Beyond Transition State Theory: Accurate Description of Nuclear Quantum Effects on the Rate and Equilibrium Constants of Chemical Reactions Using Feynman Path Integrals
}

\author{
Jiří Vaníček*
}

\begin{abstract}
Nuclear tunneling and other nuclear quantum effects have been shown to play a significant role in molecules as large as enzymes even at physiological temperatures. I discuss how these quantum phenomena can be accounted for rigorously using Feynman path integrals in calculations of the equilibrium and kinetic isotope effects as well as of the temperature dependence of the rate constant. Because these calculations are extremely computationally demanding, special attention is devoted to increasing the computational efficiency by orders of magnitude by employing efficient path integral estimators.
\end{abstract}

Keywords: Equilibrium isotope effect · Kinetic isotope effect · Path integral estimator · Quantum instanton approximation · Quantum transition state theory

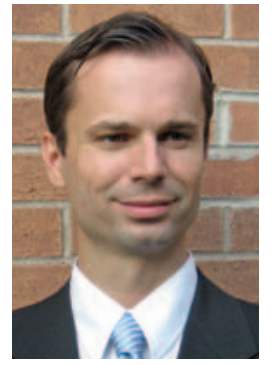

\section{Introduction}

The study of kinetics and thermodynamics of chemical reactions is a large field requiring many different experimental and theoretical techniques. Reactions between small molecules at low temperatures follow strictly laws of quantum mechanics while biochemical reactions at physiological temperatures usually abide by the laws of classical mechanics. One of the advantages of working in a theoretical laboratory is that we are not constrained by a highly specialized experimental setup and hence can study the full range of processes. On one hand, we develop new quantum and semiclassical methods to solve the timedependent Schrödinger equation, in order to study coherent quantum dynamics of small molecules ${ }^{[1-3]}$ with applications, e.g. in ultrafast electronic spectroscopy. ${ }^{[4]}$ On the other hand, we use classical statistical

${ }^{\star}$ Correspondence: Prof. Dr. J. Vaníček Laboratory of Theoretical Physical Chemistry Institut des Sciences et Ingénierie Chimiques Ecole Polytechnique Fédérale de Lausanne Avenue Forel, $\mathrm{CH}-1015$ Lausanne

Tel.: +41216934736

Fax: +41216939755

E-mail: jiri.vanicek@epfl.ch mechanics to analyze interactions between biologically important macromolecules. Specifically, we have designed several algorithms predicting which messenger RNA molecules are targeted by microRNAs, ${ }^{[5]}$ the results of which can help understanding herpes virus latency. ${ }^{[6]}$ Here I will focus on the intermediate case of thermally activated chemical reactions in which nuclear quantum effects such as tunneling and zero-point-energy effects are important, but the coherence effects are lost due to thermal averaging.

Because of the central role played by the equilibrium and rate constants in physical chemistry, many approximations for these quantities have been developed over time. The most often used approximations for the rate constant are the Arrhenius equation and transition state theory (TST). As it turns out, both of these treat nuclei essentially (i.e. up to ad hoc corrections mentioned below) as classical particles. While this often is an appropriate point of view, it fails completely when hydrogen is involved in bond breaking or formation. Indeed, strong nuclear quantum effects have been observed in many chemical reactions and recently even in quite a few enzymatic reactions. ${ }^{[7-10]}$ In order to include the quantum effects, several socalled quantum transition state theories (QTSTs) ${ }^{[11-15]}$ have been developed, the quantum instanton approximation being one of them. ${ }^{[16]}$

The importance of nuclear quantum effects is detected experimentally by mea- suring the equilibrium and kinetic isotope effects as well as the temperature dependence of the rate constant. Below I will discuss several theoretical methods for computing these quantities rigorously, i.e. methods that include nuclear quantum effects such as the zero-point energy and tunneling, and that do not assume separability of rotational and vibrational degrees of freedom or the harmonic approximation for vibrations. The improvements thus achieved will be demonstrated on three examples of hydrogen transfer reactions: (i) the simplest activated chemical reaction, $\mathrm{H}+\mathrm{H}_{2} \rightarrow \mathrm{H}_{2}+\mathrm{H}$, for which the exact quantum calculation is feasible, (ii) the [1,5]-sigmatropic hydrogen shift in (3Z)-penta-1,3-diene [compound $\mathbf{1}$, see Fig. 1(a)], ${ }^{[17]}$ and (iii) the hydrogen transfer in a much larger molecule (with 48 atoms), namely in the 2,4,6,7,9-pentamethyl-5-methylene-11,11a-dihydro- $12 \mathrm{H}$ naphtacene [compound 2, see Fig. 1(b)]. ${ }^{[18]}$

\section{Equilibrium Isotope Effects}

Let us first consider the equilibrium isotope effect (EIE), defined generally as

$$
\mathrm{EIE}=K_{l} / K_{h}
$$

where $K_{l}$ and $K_{h}$ are the equilibrium constants for the reaction with the lighter and heavier isotopolog, respectively. We shall 
(a)

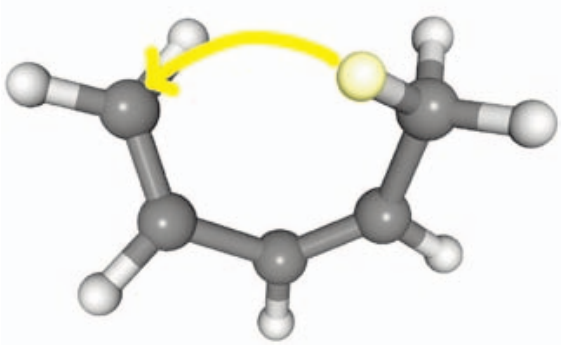

(b)

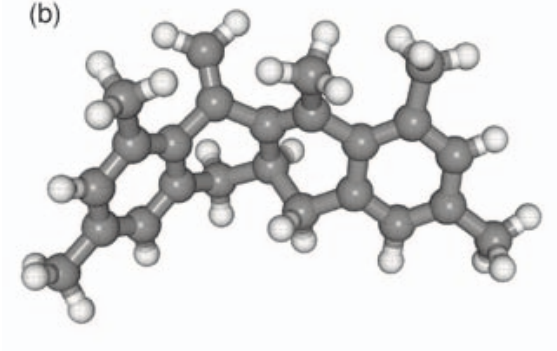

Fig. 1. (a) The [1,5] sigmatropic hydrogen shift in (3Z)-penta-1,3-diene (1). The reactant and product differ by substitution of some hydrogens by deuteriums. (b) Global minimum of 2,4,6,7,9-pentamethyl-5-methylene-11,11adihydro-12H-naphtacene (2).

consider an interesting special case - the equilibrium ratio of two isotopomers (i.e. isotopologs with the same total weight) in which the EIE is actually equal to the equilibrium constant of the isotopomerization reaction,

$$
\mathrm{EIE}=K=\frac{[p]}{[r]}
$$

where $p$ and $r$ denote the product and reactant, respectively. The equilibrium constant is given exactly in terms partition functions as $K=Q_{p} / Q_{r}$. Unfortunately, the quantum partition functions can be computed directly only for very small molecules. Therefore several basic approximations have been used. The leading contribution to the EIE (2) is the ratio of symmetry numbers of the reactant and product, $\mathrm{EIE} \approx \mathrm{EIE}_{\mathrm{sym}}=s_{r} / s_{p}$. Beyond that, one usually assumes separability of the rotations and vibrations, rigid rotor approximation for the rotations, and a harmonic approximation for the vibrations. Denoting these three approximations together simply as the harmonic approximation (HA), the EIE can be computed analytically as $\mathrm{EIE} \approx \mathrm{EIE}_{\text {sym }} \mathrm{EIE}_{\mathrm{HA}}$ where $\mathrm{EIE}_{\mathrm{HA}}$ is the ratio of quantum partition functions for harmonic oscillators and for rigid rotators whose parameters are obtained from the Hessian of the potential energy surface at the equilibrium structure. In order to include the quantum effects rigorously without any of these approximations, in ref. [19], a thermodynamic integration (TI) with respect to the mass was used to compute the $\mathrm{EIE}(2)$ as $\mathrm{EIE} \approx \mathrm{EIE}_{\mathrm{sym}} \mathrm{EIE}_{\mathrm{TI}}$

$$
\mathrm{EIE}_{\mathrm{TI}}=\exp \left[-\beta \int_{0}^{1} d \lambda \frac{d F(\lambda)}{d \lambda}\right]
$$

where $\beta=1 /\left(k_{B} \mathrm{~T}\right), F=-\beta^{-1} \ln Q$ is the free energy and $\lambda \in[0,1]$ is a parameter providing a smooth transition between the two isotopomers. This can be accomplished e.g. by linear interpolation between masses of all corresponding atoms in the molecule according to the equation

$$
m_{i}(\lambda)=(1-\lambda) m_{r, i}+\lambda m_{p, i}
$$

Unlike the partition function, the derivative $d F / d \lambda$ can be computed directly with a path integral simulation.

Before explaining the path integral procedure in detail, let us examine the results in Table 1, comparing the experimental equilibrium fractions of three isotopomers of compound $\mathbf{2}$ with the corresponding fractions calculated with three different methods. The table shows that while the HA approximation corrects the symmetry ratio in the right direction, the path integral result is closer to the experiment. Although the deviation of the experimental ratio from the symmetry ratio is quite small, it can be significant in some situations. In any case, below we will see that the nuclear quantum effects are much stronger for the kinetic isotope effect and the temperature dependence of the rate constant.

Table 1: Equilibrium fractions of three

\begin{tabular}{|c|c|c|c|}
\hline Isotopomer & $2-1,1-d_{2}$ & $2-5,5-d_{2}$ & $2-1,5-d_{2}$ \\
\hline Symmetry & 0.100 & 0.300 & 0.600 \\
\hline $\begin{array}{l}\text { Harmonic } \\
\text { approx. } .^{[19]}\end{array}$ & 0.095 & 0.312 & 0.593 \\
\hline Path integral| ${ }^{[19]}$ & 0.096 & 0.310 & 0.594 \\
\hline Experiment ${ }^{[18]}$ & 0.098 & 0.308 & 0.594 \\
\hline
\end{tabular}
isotopomers of compound 2 [see Fig. 1(b)] at $441.05 \mathrm{~K}$.

\section{Path Integral Evaluation}

How does one evaluate expression (3) with the Feynman path integrals (PIs)? The partition function, defined as

$$
Q(\beta)=\sum_{n} \exp \left(-\beta \varepsilon_{n}\right)
$$

is a central quantity in statistical thermodynamics. In this sum, $\varepsilon_{n}$ is the energy of the $n$th eigenstate. Any thermodynamic quantity such as the thermal energy or heat capacity can be expressed from the partition function if the latter is known explicitly. For a molecule with $D$ degrees of freedom, the PI representation of the partition function $Q$ is given by $Q=\lim _{P \rightarrow \infty} Q_{p}$ with

$$
Q_{P}(\beta)=C \int d \mathbf{r}^{(1)} \cdots \int d \mathbf{r}^{(P)} \exp \left[-\beta \Phi_{P}\left(\left\{\mathbf{r}^{(s)}\right\}\right)\right] .
$$

In the above equation,

$C \equiv\left(\frac{P}{2 \pi \hbar^{2} \beta^{2}}\right)^{D P / 2} \prod_{i=1}^{D} m_{i}^{P / 2}$

is a multiplicative factor, $P$ is the number of quantum replicas of the system (also called imaginary time slices), and $\mathbf{r}^{(s)} \equiv$ $\left(r_{1}^{(s)}, \ldots, r_{D}{ }^{(s)}\right)$ are the $D$ coordinates of the $s$ th replica. Finally, $\Phi_{p}\left(\left\{\mathbf{r}^{(s)}\right\}\right)$ is the effective potential given by

$$
\begin{aligned}
\Phi_{P}\left(\left\{\mathbf{r}^{(s)}\right\}\right) & =\frac{P}{2 \hbar^{2} \beta^{2}} \sum_{i=1}^{D} m_{i} \sum_{s=1}^{P}\left(r_{i}^{(s)}-r_{i}^{(s-1)}\right)^{2} \\
& +\frac{1}{P} \sum_{s=1}^{P} V\left(\mathbf{r}^{(s)}\right)
\end{aligned}
$$

with $\mathbf{r}^{(0)} \equiv \mathbf{r}^{(P)}$ and $\left\{\mathbf{r}^{(s)}\right\}$ a shorthand for $\left\{\mathbf{r}^{(1)}, \mathbf{r}^{(2)}, \ldots, \mathbf{r}^{(P)}\right\}$. The effective potential can be interpreted as a classical potential of a polymer chain: the quantum nature of the original molecule is described by the 'harmonic bonds' between the replicas (i.e. monomers) in a classical polymer chain with $P D$ degrees of freedom.

From expressions (5) and (6) it follows that for $P=1$ one obtains the classical partition function and hence classical thermodynamics. The exact quantum partition function and quantum thermodynamics are obtained in the limit $P \rightarrow \infty$, but in practice it often suffices to take quite a small value of $P$ to obtain accurate quantum results.

The thermal average $A(\beta)$ of a physical observable $A$ such as the thermal energy is computed as the PI average as in

$$
A_{P}(\beta)=\left\langle A_{P}\left(\left\{\mathbf{r}^{(s)}\right\}\right)\right\rangle_{\rho_{P}}
$$

where $\langle\cdot\rangle_{P p}$ denotes a weighted average over PI configurations. The reactant weight function is given by $\rho_{x, P}=\exp (-\beta$ $\left.\Phi_{P}\right)$ and the function $A_{P}\left(\left\{\mathbf{r}^{(s)}\right\}\right)$ is called a PI estimator for $A(\beta)$.

In practice, there exist two main strategies for evaluating the PI average (7): the PI molecular dynamics (PIMD) ${ }^{[20]}$ or PI Monte Carlo (PIMC) ${ }^{[21]}$ techniques. The results in Table $1^{[19]}$ were obtained with a PIMD implementation in the molecular dynamics package Amber 10, while the results for the KIE and the temperature dependence of the rate constant, to be discussed below, were obtained with an in- 
house PIMC code (using the Metropolis algorithm).

\section{Efficient Estimators}

The PI estimator for a given quantity turns out not to be unique. The art of PI simulations therefore lies in finding the optimal estimator, i.e. an estimator that has the smallest statistical error for a given simulation length. The simplest estimator for $d F(\lambda) / d \lambda$ is the so-called thermodynamic estimator (TE), obtained directly by substituting the PI expression (5) into Eqn. (3), giving ${ }^{[22]}$

$$
\begin{gathered}
\left(\frac{d F(\lambda)}{d \lambda}\right)_{P, \mathrm{TE}}=-\frac{P}{2 \beta} \sum_{i=1}^{D} \frac{d m_{i} / d \lambda}{m_{i}} \times \\
\times\left[1-\frac{m_{i}}{\beta \hbar^{2}} \sum_{s=1}^{P}\left(r_{i}^{(s)}-r_{i}^{(s-1)}\right)^{2}\right] .
\end{gathered}
$$

Applying a couple of tricks (namely, subtracting the centroid coordinate and mass-scaling the Cartesian coordinates) to the PI (5) yields the centroid virial estimator (CVE), $[19,23]$

$$
\begin{aligned}
& \left(\frac{d F(\lambda)}{d \lambda}\right)_{P, \mathrm{CVE}}=-\sum_{i=1}^{D} \frac{d m_{i} / d \lambda}{m_{i}} \times \\
& \times\left[\frac{1}{2 \beta}+\frac{1}{2 P} \sum_{s=1}^{P}\left(r_{i}^{(s)}-r_{i}^{(C)}\right) \frac{\partial V}{\partial r_{i}}\left(\mathbf{r}^{(s)}\right)\right]
\end{aligned}
$$

This form of the CVE is suitable for PIMD simulations. An alternative form, based on evaluating the $d F((\lambda) / d \lambda$ derivative by finite difference, is suitable for PIMC simulations in which gradients of $V$ are unavailable. ${ }^{[19,23]}$

While the statistical error of the TE grows with $P$, the statistical error of the CVE is independent of $P$. One can therefore reach the quantum limit (requiring a large $P$ ) in a much shorter simulation with the CVE. Fig. 2 compares the two estimators used in the calculation of the EIE in pentadiene. ${ }^{[19]}$ While the converged estimators agree [see Fig. 2(a)] and both require $P \geq 32$ to reach the quantum limit, Fig. 2(b) shows that for $P=64$ and a constant simulation length, the error of the CVE is ten times smaller than the error of the TE. This implies that a hundred times
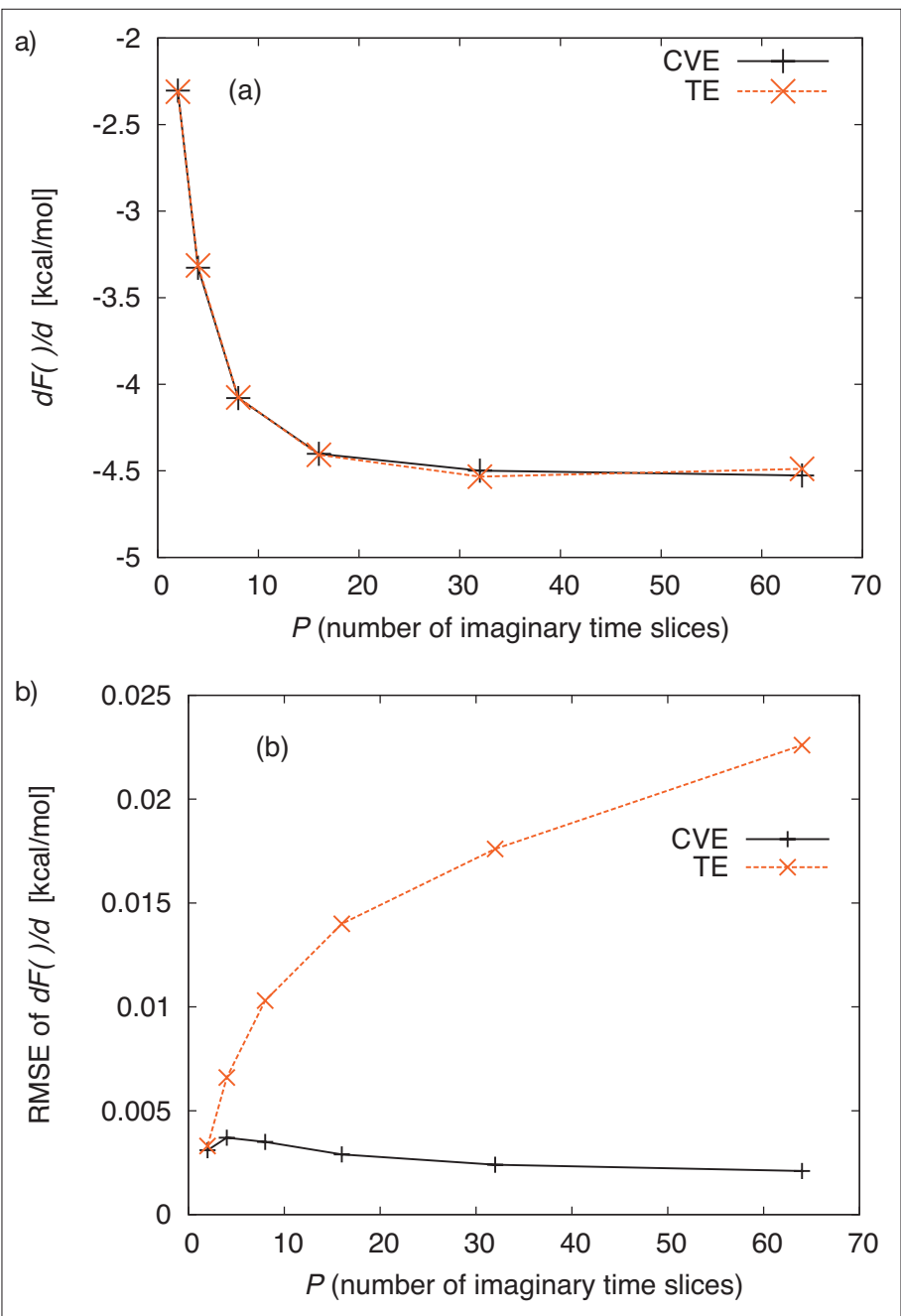

Fig. 2. (a) Dependence of the quantum free energy derivative (computed with the CVE and TE) in compound 1 on the number of replicas $P$ in the path integral. The quantum limit is reached for $P \rightarrow$ $\infty$. (b) Dependence of the root mean square error (RMSE) of the CVE and TE on $P$ for a given simulation length. For $P=64$, the RMSE of the CVE is about ten times smaller than the RMSE of the $T E$, resulting in a hundred times faster convergence of simulation. shorter simulation (!) is needed to obtain a prescribed convergence if the CVE is used instead of the TE.

\section{Kinetic Isotope Effects and the Temperature Dependence of the Rate Constant}

Having discussed in detail the PI calculation of the EIE, we are prepared for the somewhat more involved quantum treatment of chemical kinetics. The KIE is defined as the effect of isotopic substitution on the rate constant,

$$
\mathrm{KIE}=\frac{k_{l}}{k_{h}} .
$$

The KIE is widely used in chemical kinetics to detect nuclear tunneling and other nuclear quantum effects, as well as to distinguish between possible reaction mechanisms. Unlike the rate constant itself, the KIE depends very little on the height of the classical reaction barrier, and so the KIE can separate various effects from the dominating exponential dependence on the barrier height.

By the temperature dependence of the rate constant we simply mean the ratio $k(T)$ / $k\left(T_{0}\right)$ of the rate constant at two different temperatures. Similarly to the KIE, a curvature in this ratio (as a function of $T$ ) is a reflection of nuclear quantum effects and can discriminate between different reaction mechanisms.

In order to describe the KIE and the temperature dependence quantum mechanically, one needs to use an accurate expression for the rate constant itself. Clearly, the Arrhenius law

$$
k_{A}(T)=A e^{-E_{\text {act }} / k_{B} T}
$$

in which the temperature dependence is purely exponential, is not sufficient. However, for our purposes even the chemist's gold standard, i.e. the TST expression

$$
k_{\mathrm{TST}}(T)=\frac{k_{B} T}{h} \frac{Q^{\ddagger}}{Q_{r}} e^{-E^{\ddagger} / k_{B} T},
$$

is too crude. In fact, soon after Eyring's derivation, [24] Wigner ${ }^{[25]}$ showed that this expression is essentially classical: if the classical partition functions are substituted into the right-hand side of Eqn. (11), the Planck constant $h$ disappears. Several ad hoc corrections can be included into the TST expression (11), such as the zero-point energy correction or the Wigner tunneling correction (which is the leading order tunneling correction for an inverted harmonic barrier). ${ }^{[26]}$ In realistic molecules, in which rotations and vibrations are not separable and in which the vibrations are not exactly 
harmonic, one needs to use a more rigorous approach. Among these is a recently obtained quantum instanton (QI) approximation for the rate constant, ${ }^{[16]}$

$$
k_{\mathrm{QI}}(T)=\frac{\sqrt{\pi}}{2} \frac{\hbar}{\Delta H} \frac{C_{\mathrm{ff}}(0)}{Q_{r}},
$$

where $C_{\mathrm{ff}}(t)$ is the flux-flux correlation function and $\Delta H$ certain energy variance. ${ }^{27]}$ The QI approximation solves all of the above-mentioned shortcomings. In addition, unlike several methods that treat tunneling semiclassically, it considers all tunneling paths, and not just a single tunneling path (e.g. in the optimized multidimensional tunneling method of Truhlar and coworkers ${ }^{[28]}$ ) or a harmonic expansion about the optimal tunneling path (as in Miller's semiclassical instanton approximation $\left.{ }^{[29,30]}\right)$. To clarify the above statements, Fig. 3 shows the contour plot of a two-dimensional potential energy surface for a reaction $\mathrm{A}+\mathrm{BC} \rightarrow \mathrm{AB}+\mathrm{C}$ and displays regions of this surface that are considered in various methods. What QI approximation does not account for is recrossing from the product to the reactant region. However, recrossing (i) is essentially a classical effect, (ii) is missed in all classical and quantum TSTs, (iii) can be included in classical dynamical theories, and (iv) usually is quite small when quantum effects (in which we are interested the most) are important. ${ }^{[31]}$

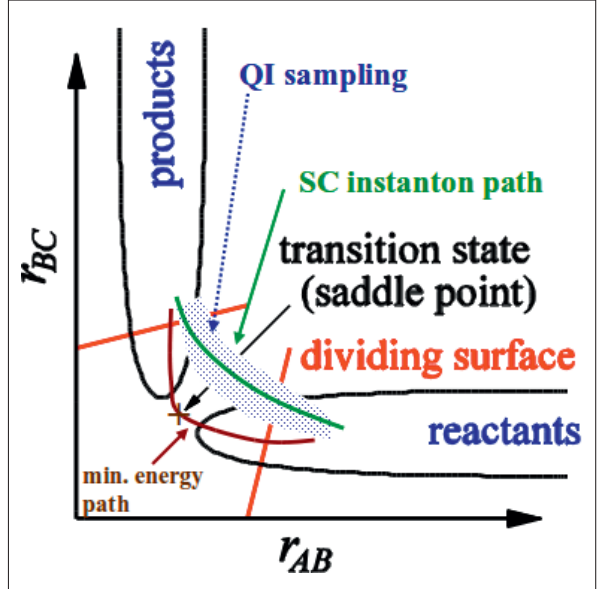

Fig. 3. Contour plot of a two-dimensional potential energy surface for the reaction $A$ $+B C \rightarrow A B+C$ and regions considered in various approximations for the reaction rate constant. The TST considers only the transition state (i.e. the saddle point of the surface) lying on the minimum energy path (MEP). The semiclassical (SC) instanton method considers a single optimal tunneling trajectory, which can be shorter than the MEP, thereby accounting for the so-called 'corner-cutting effect'.

Finally, the QI approximation considers the full ensemble of tunneling trajectories that cross the two dividing surfaces.

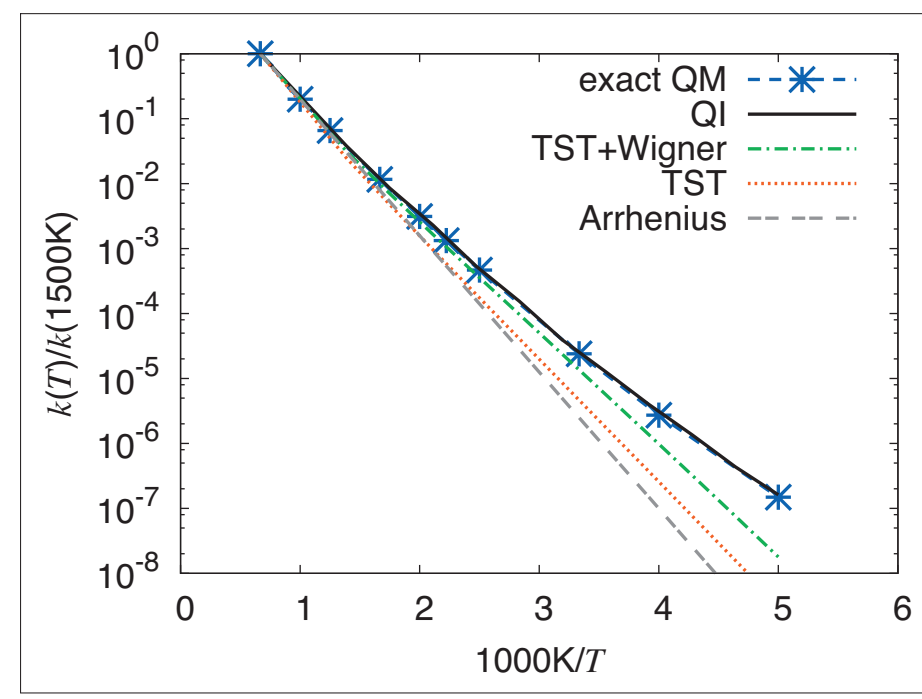

Fig. 4. Temperature dependence of the rate constant for the $\mathrm{H}+\mathrm{H}_{2} \rightarrow$ $\mathrm{H}_{2}+\mathrm{H}$ reaction: Comparison of several approximate methods $^{[33]}$ with the exact quantum mechanical (QM) calculation. ${ }^{[22]}$ The Wigner tunneling correction is insufficient to describe hydrogen tunneling in this reaction below $400 \mathrm{~K}$.

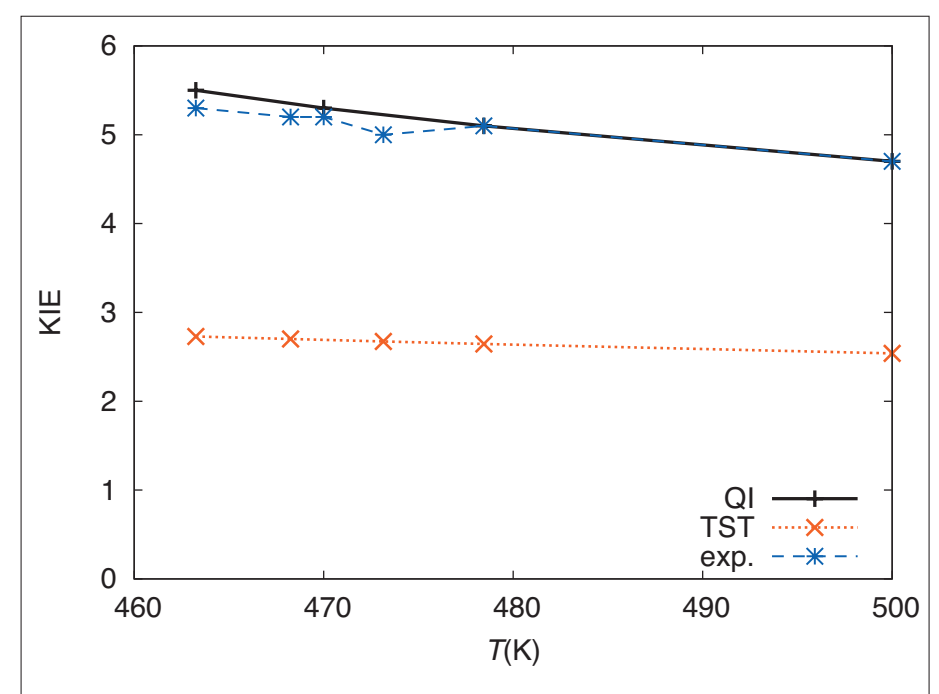

Fig. 5. Temperature dependence of the kinetic isotope effect on the $[1,5]$ sigmatropic hydrogen shift in (3Z)-penta1,3-diene [see Fig. 1(a)]. The QI and TST results were computed in ref. [32] while the experimental results are from ref. [17]. Clearly, the TST is inadequate for this reaction where hydrogen tunneling plays a major role.
It turns out to be difficult to compute the QI rate constant with PIs; this difficulty is due to the requirement of umbrella sampling between reactant and transition-state regions. ${ }^{[27]}$ We have therefore developed efficient PI methods to evaluate the $\mathrm{KIEs}^{[22,23,32]}$ and the temperature dependence of the rate constant ${ }^{[32,33]}$ directly without having to evaluate the rate constant itself first, as is done in ref. [27]. Because both the KIE and the temperature dependence $k(T) / k\left(T_{0}\right)$ are ratios of rate constants, one can employ thermodynamic integration similar to that used for the EIE. The main difference is that one needs to modify the estimators due to the constraint near the transition state and that the thermodynamic integration is with respect to the inverse temperature instead of the isotope mass in case of the temperature dependence of the rate constant. The detailed algorithms are described in refs. [19,22,23,33]. Most importantly, these references describe efficient estimators for relevant quantities, which again accelerate the calculations by orders of magnitude.
Fig. 4 shows the temperature dependence of the rate constant for the $\mathrm{H}+$ $\mathrm{H}_{2} \rightarrow \mathrm{H}_{2}+\mathrm{H}$ reaction. It shows that the TST can underestimate the rate constant by orders of magnitude. Even the Wigner tunneling correction is insufficient to describe hydrogen tunneling in this reaction below $400 \mathrm{~K}$. On the other hand, the QI approach from ref. [33] gives a very accurate temperature dependence in comparison with the exact quantum mechanical calculation from ref. [22]. Fig. 5 shows the temperature dependence of the KIE on the [1,5] sigmatropic hydrogen shift in (3Z)-penta-1,3-diene. The QI and TST results were computed in ref. [32] while the experimental results are from ref. [17]. Clearly, the TST is inadequate for this reaction, while the QI approach gives very accurate results.

\section{Discussion and Conclusion}

It was mentioned that using the efficient estimators, the quantum PI calculations can be accelerated hundred-fold at 
room temperature. Unfortunately, even after this speedup the PI simulations are extremely computationally expensive: one usually needs at least $10^{4}$ to $10^{5}$ samples, corresponding to millions of potential energy evaluations. In order to obtain results in a reasonable time, sometimes one has to sacrifice the accuracy of the electronic structure and use the less accurate force fields, semiempirical methods, or empirical valence bond potentials.

In our calculations, ${ }^{[19,32]}$ we have avoided the unnecessary loss of accuracy by using a hierarchical scheme of electronic structure methods based on the premise that the more important contributions should be treated with more accurate electronic structure methods. The barrier heights were obtained with a higher level method, the HA results with an intermediate level method, and the anharmonicity/ quantum PI corrections with a lower level method.

Despite the high cost, PI calculations of nuclear quantum effects have become increasingly popular in recent years: they have been used to find the isotope effect on the melting temperature of ice, ${ }^{[34]}$ hydrogen diffusion on a Ni surface, ${ }^{[35]}$ kinetic isotope effects on the proton transfer, ${ }^{[36]}$ or even nuclear quantum effects in enzymes. [37] When the accurate electronic structure is of greater importance than the accurate treatment of the nuclear quantum effects, one can avoid the expensive PI sampling by searching only for the optimal semiclassical tunneling trajectory, which is done in the semiclassical instanton method. This method has seen a recent revival thanks to various efficient numerical implementations. ${ }^{[38-41]}$

The work in progress in our group is on further accelerating the PI calculations (i) using high-order decomposition of the PI such as the Takahashi-Imada splitting, ${ }^{[42]}$ which would enable reaching the quantum limit with a smaller value of $P$, (ii) employing the Fourier PI sampling, [43] which would accelerate exploration of the PI configuration space, and (iii) evaluating the electronic structure much faster using graphical processing units (GPUs). [44]

In conclusion, I have discussed several ways how Feynman path integrals can be used to rigorously describe nuclear quantum effects on the equilibrium and rate constants of chemical reactions. In particular, I have addressed how such computationally demanding calculations can be accelerated with the development of efficient path integral estimators.

\section{Acknowledgments}

None of the research in the Laboratory of Theoretical Physical Chemistry would be possible without the everyday effort of $\mathrm{PhD}$ students and current and former postdoctoral associates.
I would like to thank Tomáš Zimmermann, Marcin Buchowiecki, and Sergio Gonzalez who worked on the projects discussed above. This work was supported by the Swiss National Science Foundation under the grant No. 200021124936 and by EPFL.

Received: June 9, 2011

[1] B. Li, C. Mollica, J. Vaníček, J. Chem. Phys. 2009, 131, 041101.

[2] T. Zimmermann, J. Vaníček, J. Chem. Phys. 2010, 132, 241101

[3] T. Zimmermann, J. Ruppen, B. Li, J. Vaníček, Int. J. Quantum Chem. 2010, 110, 2426.

[4] M. Wehrle, M. Šulc, J. Vaníček, Chimia 2011 65, 334.

[5] R. M. Marín, J. Vaníček, Nucleic Acids Res. 2011, 39, 19.

[6] E. Murphy, J. Vaníček, H. Robins, T. Shenk, A. J. Levine, Proc. Natl. Acad. Sci. USA 2008, 105 , 5453.

[7] A. Kohen, R. Cannio, S. Bartolucci, J. P. Klinman, Nature 1999, 399, 496.

[8] J. Basran, M. J. Sutcliffe, N. S. Scrutton, Biochemistry 1999, 38, 3218.

[9] L. Masgrau, A. Roujeinikova, L. O. Johannissen, P. Hothi, J. Basran, K. E. Ranaghan, A. J. Mulholland, M. J. Sutcliffe, N. S. Scrutton, D. Leys, Science 2006, 312, 237.

[10] D. G. Truhlar, J. L. Gao, C. Alhambra, M. Garcia-Viloca, J. Corchado, M. L. Sanchez, J. Villa, Acc. Chem. Res. 2002, 35, 341.

[11] G. A. Voth, D. Chandler, W. H. Miller, J. Chem. Phys. 1989, 91, 7749 .

[12] N. F. Hansen, H. C. Andersen, J. Phys. Chem. 1996, 100, 1137.

[13] E. Pollak, J. L. Liao, J. Chem. Phys. 1998, 108, 2733.

[14] R. Schubert, H. Waalkens, S. Wiggins, FewBody Systems 2009, 45, 203.

[15] A. Goussev, R. Schubert, H. Waalkens, S. Wiggins, J. Chem. Phys. 2010, 133, 244113.

[16] W. H. Miller, Y. Zhao, M. Ceotto, S. Yang, J. Chem. Phys. 2003, 119, 1329.

[17] W. R. Roth, J. Konig, Ann. Chemie-Justus Liebig 1966, 699, 24.

[18] W. V. Doering, E. J. Keliher, J. Am. Chem. Soc. 2007, 129, 2488

[19] T. Zimmermann, J. Vaníček, J. Chem. Phys. 2009, 131, 024111 .
[20] B. J. Berne, D. Thirumalai, Annu. Rev. Phys. Chem. 1986, 37, 401.

[21] D. M. Ceperley, Rev. Mod. Phys. 1995, 67, 279

[22] J. Vaníček, W. H. Miller, J. F. Castillo, F. J. Aoiz, J. Chem. Phys. 2005, 123, 054108 .

[23] J. Vaníček, W. H. Miller, J. Chem. Phys. 2007, 127,114309

[24] H. Eyring, J. Chem. Phys. 1935, 3, 107

[25] E. Wigner, Trans. Faraday Soc. 1938, 34, 0029.

[26] E. Wigner, Z. Phys. Chem. B-Chem. E 1932, 19 , 203.

[27] T. Yamamoto, W. H. Miller, J. Chem. Phys. 2004, 120, 3086.

[28] Y. P. Liu, G. C. Lynch, T. N. Truong, D. H. Lu, D. G. Truhlar, B. C. Garrett, J. Am. Chem. Soc. 1993, 115,2408

[29] W. H. Miller, J. Chem. Phys. 1975, 62, 1899.

[30] S. Chapman, B. C. Garrett, W. H. Miller, $J$. Chem. Phys. 1975, 63, 2710.

[31] M. Ceotto, W. H. Miller, J. Chem. Phys. 2004 $120,6356$.

[32] T. Zimmermann, J. Vaníček, J. Mol. Mod. 2010 16, 1779.

[33] M. Buchowiecki, J. Vaníček, J. Chem. Phys. 2010, 132, 194106

[34] R. Ramirez, C. P. Herrero, J. Chem. Phys. 2010 133,144511

[35] Y. Zhao, W. J. Wang, J. Chem. Phys. 2009, 130

[36] K. F. Wong, J. L. Sonnenberg, F. Paesani, T. Yamamoto, J. Vaníček, W. Zhang, H. B Schlegel, D. A. Case, T. E. Cheatham, W. H. Miller, G. A. Voth, J. Chem. Theory Comput. 2010, 6, 2566.

[37] A. Azuri, H. Engel, D. Doron, D. T. Major, $J$ Chem. Theory Comput. 2011, 7, 1273.

[38] Z. Smedarchina, W. Siebrand, A. FernandezRamos, Q. Cui, J. Am. Chem. Soc. 2003, 125, 243.

[39] J. B. Rommel, T. P. M. Goumans, J. Kastner, $J$. Chem. Theory Comput. 2011, 7, 690.

[40] S. C. Althorpe, J. Chem. Phys. 2011, 134 114104.

[41] M. Kryvohuz, J. Chem. Phys. 2011, 134, 114103

[42] M. Takahashi, M. Imada, J. Phys. Soc. Jpn. 1984, 53, 3765.

[43] J. D. Doll, J. Chem. Phys. 1984, 81, 3536.

[44] I. S. Ufimtsev, T. J. Martinez, J. Chem. Theory Comput. 2009, 5, 2619. 